\title{
Primäre Immundefekte und Autoimmunität
}

\author{
Rheumatologische Manifestationen bei pädiatrischen Patienten mit primären \\ Immundefekten - was gibt es Neues?
}

\section{Primary immunodeficiencies and autoimmunity}

\author{
Rheumatological manifestations in pediatric patients with primary immunodeficiency \\ - an update
}

Autorin

Almut Meyer-Bahlburg

Institut

Klinik und Poliklinik für Kinder und Jugendmedizin,

Pädiatrische Rheumatologie \& Immunologie,

Universitätsmedizin Greifswald

Schlüsselwörter

Primärer Immundefekt, Immundysregulation, Lympho-

proliferation, CVID

Keywords

Primary immunodeficiency, immune dysregulation, lymphoproliferation, CVID

Bibliografie

DOI https://doi.org/10.1055/a-1275-7759

arthritis + rheuma 2020; 40: 440-448

(c) 2020. Thieme. All rights reserved.

Georg Thieme Verlag KG, Rüdigerstraße 14,

70469 Stuttgart, Germany

ISSN 0176-5167

Korrespondenzadresse

Univ.-Prof. Dr. med. Almut Meyer-Bahlburg

Pädiatrische Rheumatologie und Immunologie

Universitätsmedizin Greifswald, KöR

Ferdinand-Sauerbruch-Straße

17475 Greifswald

Deutschland

almut.meyer-bahlburg@med.uni-greifswald.de

\section{ZUSAMMENFASSUNG}

Primäre Immundefekte sind seltene angeborene Erkrankungen, die lange Zeit vor allem durch eine erhöhte Infektanfälligkeit und das Auftreten schwerer und opportunistischer Infektionen charakterisiert waren. In den letzten Jahren wurden aber zunehmend primäre Immundefekte identifiziert, bei denen die Immundysregulation im Vordergrund steht und die durch Autoimmunität und Autoinflammation gekennzeichnet sind. Chronische Arthritiden und andere rheumatologische Symptome können dabei nicht nur als Komplikation der Immundefekte auftreten, sondern auch eines der charakteristischen Krankheits-definierenden Symptome darstellen. Gerade im Kindes- und Jugendalter kann die rheumatologische Manifestation zudem als erstes klinisches Symptom des zugrunde liegenden Immundefekts auftreten. Daher ist in der Kinder- und Jugendrheumatologie ein grundlegendes Wissen über primäre Immundefekte unabdingbar. Im folgenden Übersichtsartikel soll ein Überblick über primäre Immundefekte, die mit rheumatologischen Symptomen einhergehen können, gegeben werden. Ein besonderer Fokus liegt dabei auf kürzlich charakterisierten neuen Immundefekten, bei denen die Autoimmunität häufig im Vordergrund steht.

\section{ABSTRACT}

Primary immunodeficiencies are rare diseases generally characterized by increased susceptibility to infections. In contrast to this definition, over the last years several primary immunodeficiency syndromes were identified that mainly present with immune dysregulation, namely autoimmunity and autoinflammation, as major manifestations. In some cases, chronic arthritis and other rheumatological phenomena might be the most important symptom in these immunodeficiencies. Especially in children and teenagers, rheumatological symptoms can also be the first and, at time of diagnosis, only symptom of the underlying immune dysregulation. Therefore, pediatric rheumatologists should provide a basic knowledge about primary immunodeficiencies. This review comprises recent updates on primary immunodeficiencies with immune dysregulation and rheumatological manifestations. 


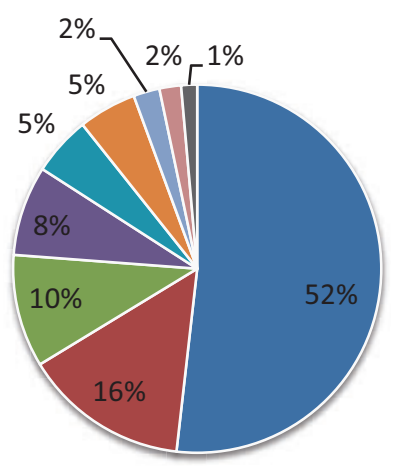

n Immundefekte mit Antikörpermangel im Vordergrund: 14506 (51,8 \%)

- Gut definierte Syndrome mit Immundefekt: 4051 (14,5\%)

- Kombinierte T- und B-Zellen Immundefekte: 2779 (9,9\%)

- Angeborene Defekte der Phagozyten: 2202 (7,9\%)

- Erkrankungen mit Immundysregulation: 1468 (5,2\%)

- Komplementdefekte: 1416 (5,1\%)

- Autoinflammatorische Erkrankungen: 635 (2,3\%)

- Abb. 1 Klassifikation und Häufigkeiten von primären Immundefekten.

Primäre Immundefekte sind seltene angeborene Erkrankungen, die häufig monogenetisch vererbt werden. Die zunehmenden Möglichkeiten der molekulargenetischen Diagnostik einschließlich des Whole-Exome-Sequencings haben in den letzten Jahren zur Identifizierung zahlreicher neuer Immundefekte geführt. Im letzten Bericht der „International Union of Immunological Societies“ (IUIS) von 2017 wurden 354 verschiedene Immundefekte aufgeführt [1]. Nach der derzeitigen Klassifikation werden Immundefekte in 8 Untergruppen eingeteilt, auch wenn in manchen Fällen eine Zuordnung schwierig ist oder eine Erkrankung mehreren Untergruppen zugeordnet werden könnte. > Abb. 1 zeigt die Klassifikation einschließlich der Häufigkeiten basierend auf Daten des europäischen Registers für primäre Immundefekte.

Lange Zeit waren primäre Immundefekte vor allem durch eine erhöhte Infektanfälligkeit und das Auftreten schwerer und opportunistischer Infektionen charakterisiert. In den letzten Jahren wurden aber zunehmend primäre Immundefekte identifiziert, bei denen die Immundysregulation im Vordergrund steht und die durch das Auftreten von Autoimmunität gekennzeichnet sind. Die häufigsten dabei auftretenden Autoimmunphänomene stellen in der Regel Zytopenien dar, wobei eine oder mehrere Zellreihen betroffen sind [2]. Rheumatologische Manifestationen können sich als chronische Arthritiden, systemischer Lupus erythematodes (SLE), Sjögren-Syndrom-ähnliche Erkrankungen, Vaskulitiden oder auch als Sklerodermie äußern. Daneben können Endokrinopathien einschließlich Erkrankungen der Schilddrüse, Typ-1-Diabetes mellitus (T1DM), Enteropathien, interstitielle Lungenerkrankungen, Glomerulonephritiden, ZNS-Erkrankungen und Hepatitiden auftreten.

In einer französischen Studie von Alain Fischer über Autoimmunphänomene bei primären Immundefekten wurde gezeigt, dass über $26 \%$ der Patienten ein oder mehrere Autoimmunsymptome aufwiesen [3]. In dieser Studie war das Auftreten einer Arthritis bei pädiatrischen Immundefekt-Patienten bis 18 Jahre gegenüber der gesunden Bevölkerung 40fach erhöht.

Im Folgenden werden verschiedene Immundefekte vorgestellt, bei denen rheumatologische Manifestationen auftreten können. Dabei wird insbesondere auf die in den letzten Jahren neu beschriebenen Immundefekte eingegangen, bei denen Autoimmunphänomene ein wichtiges Symptom darstellen, wie genetische Defekte der Signaltransduktion (NF-KB, PI3K, STAT1 und 3) oder Erkrankungen mit Immundysregulation (LRBA, CTLA4).

\section{Antikörpermangelsyndrome}

Die X-chromosomal vererbte Agammaglobulinämie (XLA, auch Morbus Bruton) wurde 1952 von dem US-Militärarzt Colonel Ogden Bruton erstmals beschrieben und stellt damit einen der ersten charakterisierten Immundefekte dar [4]. Durch eine Mutation in dem nach der Erkrankung benannten Bruton's Thyrosin Kinase Gen kommt es zu einer defekten Signaltransduktion über den B-Zell-Rezeptor und dadurch zu einem fast kompletten Fehlen der B-Zellen sowie folglich auch der Immunglobuline. Inzwischen wurden weitere genetische Defekte identifiziert, die zu einer Agammaglobulinämie führen $(\mu$ heavy chain, $\lambda 5$, Ig $\alpha$ - und Ig $\beta$-Defekte) und autosomal rezessiv vererbt werden, sodass auch Mädchen betroffen sein können [1]. Charakteristischerweise erkranken die betroffenen Kinder nach dem Nachlassen der mütterlichen Leihimmunität an rezidivierenden Infekten der Luftwege und schweren bakteriellen Infektionen. Arthritiden sind vor allem bei XLA-Patienten beschrieben, wobei gemäß einer Studie mit 36 Patienten 17\% betroffen waren [5]. Dabei reicht das Spektrum von der oligoartikulären [6] über die Enthesitis-assoziierte [7] bis hin zur erosiven Polyarthritis [8].

Der Gruppe der Hyper-IgM-Syndrome liegt ein Defekt im Immunglobulin-Klassenwechsel zu Grunde. Dadurch kann kein, bzw. kaum klassengewechseltes IgG, IgA und auch IgE gebildet werden. Stattdessen kommt es zu deutlich erhöhten IgM-Spiegel, das IgM kann jedoch auch normwertig oder sogar vermindert sein. Die Zahl der B-Zellen ist normwertig, klassengewechselte Gedächtnis-B-Zellen sind jedoch vermindert oder fehlen komplett. Auch für diese Gruppe von Immundefekten wurden inzwischen mehrere Gene identifiziert (CD40L [Typ 1], AID [Typ 2], CD40 [Typ 3], UNG [Typ 4]), die Erkrankung wird X-chromosomal (Typ 1) oder auch autosomal-rezessiv (Typ 2-4) vererbt. Typischerweise erkranken betroffene Individuen an rezidivierenden Atemwegsinfekten, aber auch opportunistischen Infektionen mit Pneumocystis jiroveci oder Kryptosporidien. Autoimmunsymptome treten besonders häufig bei dem durch CD40L-Defekt verursachten Hyper-IgM-Syndrom (Typ 1) auf. So wurde über chronische, teilweise auch erosive Arthritiden bei 11-18\% dieser Patienten berichtet [9-11]. Ein SLE hingegen wurde beim $\mathrm{Hy}$ per-IgM-Syndrom durch AID-Mutation beschrieben [12].

Der selektive IgA-Mangel ist der häufigste Immundefekt, oft jedoch asymptomatisch. Diagnose-Kriterien sind ein $\lg A<0,07 \mathrm{~g} / \mathrm{I}$ mit normwertigem IgG und IgM bei Kindern über 4 Jahren. Autoim- 
munphänomene treten beim selektiven IgA-Mangel mit einer Häufigkeit von 7-36\% auf [13]. Dabei wurde bei erwachsenen Patienten in 2-4\% eine chronische rheumatoide Arthritis und in 1-5\% ein SLE beschrieben. Auch ist im umgekehrten Fall bei der JIA eine Assoziation mit dem selektiven IgA-Mangel bekannt, der bei etwa 2-4\% der JIA-Patienten besteht [14]. Die genetische Ursache des selektiven IgA-Mangels ist weitgehend unbekannt, jedoch ist der Übergang zum sogenannten variablen Immundefekt (common variable immunodeficiency, CVID) fließend. So können in derselben Familie Mitglieder sowohl vom selektiven IgA-Mangel als auch von einem CVID betroffen sein.

Der CVID stellt den häufigsten symptomatischen humoralen Immundefekt bei Erwachsenen dar und hat einen heterogenen Ursprung. Derzeit ist bei etwa $10 \%$ der Patienten ein zugrundeliegender genetischer Defekt bekannt. Autoimmunsymptome sind mit 23 \% so häufig [15], dass sie inzwischen ein Diagnosekriterium darstellen. Nach den Kriterien der European Society of Primary Immunodeficiendy (ESID) kann ein CVID bei einem Mangel von IgG und IgA, fehlenden spezifischen Antikörpern oder verminderten Gedächtnis-B-Zellen sowie entsprechenden klinischen Symptomen, darunter Autoimmunphänomene, diagnostiziert werden (siehe Kasten „Klinische Kriterien für einen CVID“) http://esid.org/Working-Parties/Registry/Diagnosis-criteria). Seit längerem bekannte monogenetische Ursachen liegen in Molekülen des B-Zell-Rezeptors (CD19, CD20, CD21, CD81 [16-20]), einem B-Zell-Überlebensfaktor bzw. dessen Rezeptoren (B cell activation factor of the TNF family [BAFF], BAFF-R und TACI [21, 22]) oder ko-stimulatorischen Molekülen in der Keimzentrumsreaktion (CD27, ICOS [20]). In den letzten Jahren wurden einige neue genetische Defekte bei CVID-Patienten gefunden, auf die hier näher eingegangen werden soll.

\section{NF-KB 1 und 2 Loss-of-function Mutationen}

Zur Familie der NF-кB-Transkriptionsfaktoren zählen 5 Mitglieder: NF-кB1, NF-кB2, RelA, RelB und c-Rel. Der NF-кB Signalweg ist in zahlreichen zellulären Prozessen involviert, in B-Lymphozyten zählen dazu insbesondere die Zell-Reifung und -Differenzierung, das Überleben sowie der Immunglobulin-Klassenwechsel. In einer Europäischen Kohorte von 390 CVID Patienten war eine Loss-of-function Mutation im NF-kB1-Gen mit 4\% die häufigste monogenetische Ursache der Erkrankung [23]. Die Mutation trat sowohl sporadisch als auch familiär auf. Neben einer Hypogammaglobulinämie und einer gestörten B-Zell-Differenzierung bestand bei $24 \%$ der Patienten eine Lymphadenopathie, bei $48 \%$ eine Splenomegalie und bei $48 \%$ Autoimmunsymptome, darunter Polyarthritiden. Auch Mutationen in NF-kB2 können zu einem CVID-Phänotyp mit Autoimmunität führen. Allerdings sind dafür bisher keine rheumatologischen Manifestationen beschrieben worden, lediglich eine Alopezie sowie eine Psoriasis-artige Dermatitis wurden aufgeführt [24].

\section{Aktivierte PI3 Kinase delta Syndrom (APDS)}

Die Phosphatidylinositol 3 Kinase delta (PI3Kס) stellt eine wichtige Kinase in der Signaltransduktionskaskade nach Stimulation über den B- und T-Zell-Rezeptor sowie verschiedenen anderen Rezeptoren (z. B. TLR, Kostimulatoren) dar und hat damit einen entscheidenden Einfluss auf die Lymphozytendifferenzierung und -funk-

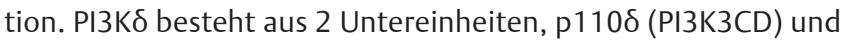
p85 $\alpha$ (PI3K3R1). Heterozygote Gain-of-function (GOF) Mutationen

\section{KLINISCHE KRITERIEN FÜR EINEN CVID}

Mindestens eines der folgenden Symptome:

- Erhöhte Infektanfälligkeit

- Autoimmunsymptome

- Granulomatöse Erkrankungen

- Unerklärliche polyklonale Lymphoproliferation

- Andere Familienmitglieder mit Antikörper-Defizienzen

UND deutlich vermindertes IgG und IgA mit oder ohne Verminderung von IgM (mindestens zwei Bestimmungen; <2SD unterhalb des altersabhängen Normbereichs)

UND mindestens einer der folgenden Befunde:

- verminderte Impfantikörper-Titer (und/oder fehlende Isoagglutinine); fehlende protektive Antikörpertiter trotz Impfung soweit definiert

- niedrige Anzahl an klassengewechselten Gedächtnis-B-Zellen (<70\% des altersabhängigen Normbereichs)

UND Auschluss sekundärer Ursachen einer Hypogammaglobulinämie (Infektion, Eiweißverlust, Medikamente, Malignitäten)

UND Diagnose nach Beendigung des 4. Lebensjahres (auch wenn Symptome schon früher aufgetreten sind)

UND kein Hinweis auf tiefergehende T-Zell-Störung, manifest, sobald 2 der folgenden Kriterien vorliegen:

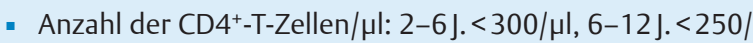
$\mu \mathrm{l},>12 \mathrm{~J} .<200 / \mu \mathrm{l}$

- \% naive CD4+-T-Zellen von Gesamt-CD4 ${ }^{+}$-T-Zellen: $2-6$ J. $<25 \%, 6-16$ J. $<20 \%,>16$ J. $<10 \%$

- fehlende T-Zell Proliferation

in $\mathrm{p} 110 \delta$ und $\mathrm{p} 85 \alpha$ führen zum aktivierten PI3 Kinase delta Syndrom 1 und 2 (APDS 1 und 2) [25-27]. Klinisch können sich diese Patienten mit einem CVID-ähnlichen Krankheitsbild präsentieren. Jedoch ist die klinische Symptomatik sehr heterogen, sodass sich diese Erkrankung auch unter anderen klinischen Diagnosen wie Hyper-IgM-Syndrom, kombinierter Immunfekt (Combined Immunodeficiency, CID), IgG-Subklassen-Defekt oder XLA verbergen kann, wie in einem kürzlich veröffentlichten Review gezeigt wurde [27]. Viele dieser Patienten zeigen neben Störungen in der B-Zell-Differenzierung auch eine gestörte T-Zell-Funktion. Da bei einem CVID definitionsgemäß eine Störung der T-Zellen ein Ausschlusskriterium darstellt (siehe Kasten „Klinische Kriterien für einen CVID“), könnte die Erkrankung eher der CID-Gruppe zugeordnet werden. Gegenwärtig wird das APDS aber in der IUIS-Klassifikation unter den humoralen Immundefekten aufgeführt [1].

APDS-Patienten präsentieren sich in der Regel mit einer mäßigen Hypogammaglobulinämie (dabei besteht gelegentlich ein erhöhter IgM-Spiegel, was zur Diagnose eines Hyper-IgM-Syndroms führen kann), rezidivierenden Infekten der Atemwege, Lymphoproliferation mit teilweise sehr stark ausgeprägter Lymphadenopathie (75-89\%) und Splenomegalie. Autoimmunphänomene 
treten in etwa 17-35\% der APDS-Patienten auf, darunter werden neben Zytopenien und Glomerulonephritiden auch chronische Arthritiden beschrieben: In einer Kohorte von 53 APDS-Patienten bestand bei 2 Patienten eine seronegative Arthritis [28], in einer anderen Studie mit 36 APDS-Patienten litten ebenfalls $2 \mathrm{~Pa}$ tienten an einer chronischen Arthritis [26]. Detailliertere Angaben zu den Arthritiden sind den Veröffentlichungen jedoch nicht zu entnehmen.

\section{Erkrankungen mit Immundysregulation}

Bei der Gruppe der Erkrankungen mit Immundysregulation stehen Autoimmunerkrankungen im Vordergrund des klinischen Erscheinungsbildes. Zeichen eines Immundefektes im Sinne von rezidivierenden Infekten und opportunistischen Infektionen sind fakultativ und treten, abhängig von dem zugrunde liegenden Defekt, nur in einigen Fällen auf. Den Prototyp einer monogenetischen Erkrankung mit Immundysregulation stellt das IPEX-Syndrom (Immundysregulation, Polyendokrinophathie, Enteropathie, X-chromosomale Vererbung) dar, das durch eine Mutation im FOXP3-Gen verursacht wird. Dadurch besteht ein funktioneller Defekt, bzw. Fehlen der regulatorischen T-Zellen. Bei den betroffenen Patienten kommt es, häufig schon im Säuglingsalter beginnend, zum Auftreten einer ausgeprägten Enterokolitis, eines Ekzems, eines Diabetes mellitus Typ 1, einer Thyreoiditis, einer hämolytischen Anämie, anderer Zytopenien oder einer Glomerulonephritis. In einer großen Studie mit 96 IPEX-Patienten wurden zudem bei 8 Patienten auch Arthritiden beschrieben [29], in einer anderen Studie mit 88 IPEX-Patienten litten $9 \%$ an einer Arthritis oder Vaskulitis [30].

Das IPEX-ähnliche Syndrom steht für eine Gruppe von Erkrankungen, bei denen die Patienten an vergleichbaren Symptomen wie IPEX-Patienten erkranken, aber keine Mutation im FOXP3-Gen gefunden werden konnte. Da auch hierbei Defekte der regulatorischen T-Zellen bestehen, entstand der Begriff der „Tregopathien“ [31]. Inzwischen wurden mehrere zugrunde liegende monogenetische Erkrankungen für Tregopathien identifiziert, von denen einige hier näher dargestellt werden sollen, da auch rheumatologische Manifestationen auftreten.

\section{CTLA-4- und LRBA-Defizienz}

Cytotoxic-T-Lymphocyte-antigen 4 (CTLA-4) ist ein inhibitorisches Oberflächenmolekül, das auf T-Zellen, insbesondere auch regulatorischen T-Zellen exprimiert ist [30]. CLTA-4 bindet an CD80 und CD86 auf Antigen-präsentierenden Zellen, und verhindert so eine Interaktion mit CD28 auf Effektor-T-Zellen, die dieses als 2. Signal zur weiteren Aktivierung benötigen. Eine Haploinsuffizienz von CTLA-4 führt daher zu einer vermehrten Stimulation von Lymphozyten, was in einer Immundysregulation resultiert. Neben rezidivierenden Infekten der Atemwege und einer variabel ausgeprägten Hypogammaglobulinämie können Zytopenien, Lymphoproliferation mit Splenomegalie, Hepatomegalie und Lymphadenopathie, Enteropathien, atopische Dermatitis, Autoimmunenzephalitis und -thyreoiditis, aber auch Arthritiden (rheumatoide Arthritis, Psoriasisarthritis) auftreten [32, 33].

Auch die LRBA-Defizienz ist durch eine variable Hypogammaglobulinämie, rezidivierende Atemwegsinfekte und Immundysre- gulation gekennzeichnet [34]. Das LPS responsive beige-like anchor (LRBA) Protein bindet an das zytoplasmatische Ende von CTLA-4 und verhindert so dessen lysosomalen Abbau. Ist LRBA vermindert vorhanden, wird CTLA-4 entsprechend vermehrt degradiert und es resultiert eine Immundysregulation ähnlich der CTLA-4 Defizienz [35]. Die Erkrankung ist durch eine homozygote oder compound heterozygote Mutationen verursacht. Auch hier können Arthritiden auftreten, welche schon im Kindesalter beschrieben sind [36].

\section{STAT1- und STAT3 Gain-of-Function Mutationen}

Der JAK-STAT-Signalweg ist ein wichtiger Bestandteil der Signaltransduktionskaskade zahlreicher Zytokinrezeptoren (IL-6, IL10, IL-12, IL-22, IL-23, INFy, INF $\alpha$ ) [37]. Zunehmend wird eine therapeutische Inhibition verschiedener Januskinasen (JAK 1-3, TYK2) bei rheumatischen Erkrankungen erfolgreich angewendet. In der Signaltransduktionskaskade werden nach den Januskinasen die STAT-Transkriptionsfaktoren aktiviert. Aus der Familie der Signal Transducers and Activators of Transcription (STAT)-Proteine sind derzeit 7 Mitglieder bekannt: STAT1-4, STAT5a und b, STAT6 [38]. Mutationen in diesen Transkriptionsfaktoren können sowohl zu einer Unterfunktion (Loss-of-Function, LOF) als auch Überfunktion (Gain-of-Function, GOF) von STATs führen. So resultiert eine LOF-Mutation in STAT3 im autosomal-dominant vererbten Immundefekt Hyper-IgE-Syndrom, welches klinisch durch rezidivierende Hautabszesse, pulmonale Infektionen, ausgeprägtes Ekzem, Eosinophilie und typischen Habitus charakterisiert ist [39]. Im Gegensatz dazu führt eine autosomal-dominante GOF-Mutation in STAT3 zur Immundysregulation mit Lymphoproliferation und Autoimmunität. In einer Veröffentlichung von Haapaniemi et al. mit 19 STAT3-GOF-Patienten wurden folgende Autoimmunphänomene beschrieben: early-onset Typ 1 Diabetes mellitus (6/19), Autoimmun-Zytopenien (14/19), Lymphadenopathie (11/19), Lymphoproliferation 10/19), Enteropathie (6/19), Zöliakie (2/19), unspezifische Kolitis (1/19), interstitielle Lungenerkrankung (1/19), Hautmanifestationen (Ekzem, Alopezie, Dermatitis 9/19), Arthritis (3/19) und Uveitis (1/19) [40]. In einer 2. Kohorte mit 13 STAT3-GOF-Patienten aus 10 Familien litten 2 Patienten an einer Polyarthritis, beide im Kleinkindalter beginnend. Bei einem dieser beiden Patienten bestand zudem eine Sklerodermie [41].

Auch bei STAT1 gibt es sowohl LOF- als auch GOF-Mutationen, beide werden autosomal dominant vererbt. Eine STAT1-LOF-Mutation war eine der ersten identifizierten monogenetischen Ursachen für eine erhöhte Suszeptibilität für Mykobakterien [37]. Die betroffenen Individuen leiden unter atypischen Mykobakteriosen und anderen viralen Infekten. Im Gegensatz dazu ist STAT1-GOF-Patienten eine chronisch mukokutane Candidiasis (CMC) charakteristisch. Dabei besteht in der Regel eine ausgeprägte Candidiasis der Schleimhäute, der Haut und auch der Nägel. Darüber hinaus leiden die betroffenen Individuen häufig an bakteriellen Infekten der Atemwege und der Haut (in 36 \% durch S. aureus), viralen Infektionen, insbesondere mit Herpesviren und auch invasiven Pilzinfektionen (v. a. Candida spp) [42]. In einer großen Kohorte mit 274 STAT1-GOF-Patienten wurden zudem bei $37 \%$ der Patienten verschiedene Autoimmunphänomene beschrieben: Hypothereose (22\%), Diabetes mellitus Typ 1 (4\%), Zytopenien (4\%), und in $2 \%$ ein systemischer Lupus erythematodes mit Vaskulitis, Serositis und 
Anti-phospholipid Syndrom. Ein Patient litt zudem unter einer Sklerodermie und bei 28 Patienten wurde eine Hautbeteiligung (Vitiligo, Alopezie, Psoriasis) beschrieben.

\section{Immundefekte anderer Gruppen}

Zahlreiche weitere Immundefekte können mit Autoimmunphänomenen einhergehen. In der Gruppe der gut definierten Syndrome mit Immundefekt ist die 22q11-Mikrodeletion zu erwähnen. Hier tritt in etwa $2 \%$ der Patienten eine Polyarthritis, meist im Kindesalter beginnend, auf $[43,44]$. In diesen Fällen besteht interessanterweise häufig zusätzlich ein selektiver IgA-Mangel.

Auch das X-chromosomal vererbte Wiskott-Aldrich-Syndrom zählt zu dieser Gruppe und kann klinisch durch die typische Trias aus rezidivierenden Infekten, v. a. mit bekapselten Erregern, mikrozytärer Thrombozytopenie und Ekzem in Erscheinung treten. In Bezug auf Autoimmunphänomene wurde über Zytopenien, Glomerulonephritiden, Myositiden, Vaskulitiden, Hepatitiden und chronische Arthritiden berichtet [45].

Die septische Granulomatose ist durch Mutationen in Genen für die NADPH-Oxidase bedingt. Dadurch kommt es zu einer verminderten Bildung von Sauerstoffradikalen und somit funktionellen Störung der Granulozyten. Abhängig von der Mutation wird die Erkrankung X-chromosomal oder autosomal-rezessiv vererbt. Neben rezidivierenden bakteriellen Infekten mit Abszessbildung kommt es häufiger zum Auftreten einer chronischen Kolitis. Aber auch eine chronische Arthritis wurde bei einem Kind mit septischer Granulomatose beschrieben [46]. Bei der X-chromosomal vererbten Form der septischen Granulomatose (gp91-Mutation) treten Autoimmunphänomene insbesondere bei den weiblichen Überträgerinnen auf. In einer prospektiven Studie mit 19 Überträgerinnen traten im Verlauf bei 58\% SLE typische Hauterscheinungen, bei $42 \%$ orale Ulzera, bei $37 \%$ Gelenkschmerzen und bei $27 \%$ positive ANA-Titer auf [47].

Komplement-Defekte können bekanntermaßen zu einem SLE führen [48]. Auch das breite Spektrum der Autoinflammation mit dem Auftreten von Autoimmunsymptomen ist Rheumatologen im Allgemeinen gut bekannt und wird daher hier nicht weiter aufgeführt. \ Tab. 1 zeigt eine Übersicht von rheumatologischen Manifestationen bei verschiedenen Immundefekten.

\section{Diagnostik bei V.a. primäre Immundefekte}

Grundsätzlich sollte bei jeder Erstvorstellung wegen rheumatologischer Beschwerden das Vorliegen eines Immundefektes zumindest erwogen, bzw. ausgeschlossen werden. Das ist in der Regel sicherlich gängige Praxis in kinderrheumatologischen Ambulanzen. Letztlich handelt es sich bei der JIA ja um eine klinische Ausschlussdiagnose, wozu auch immer der Ausschluss eines zugrunde liegenden Immundefektes als Ursache einer Arthritis gehört. Gerade bei Kindern können rheumatische Beschwerden das Erstsymptom einer Immundysregulation darstellen, weshalb dieser Aspekt zunehmend mehr Beachtung finden sollte.

Anamnestisch muss abgeklärt werden, ob rezidivierende Infekte auftreten oder wiederholt schwere (bakterielle Pneumonie, Sepsis, Meningitis, Osteomyelitis) oder opportunistische Infektionen aufgetreten sind. Hier sind ggf. auch rekurrierende atypische Mykobakteriosen zu erfragen. In Bezug auf die Familienanamne- se sollte neben anderen betroffenen Familienmitgliedern auch nach unklaren Todesfällen gefragt werden. Zudem sind die typischen 12 Warnzeichen von primären Immundefekten zu beachten (https://www.immundefekt.de/12-warnzeichen).

Eine Gedeihstörung wird durch die übliche Aufnahme der Körpermaße erfasst. Bei der klinischen Untersuchung können Exantheme oder Ekzeme, eine Candidiasis, eine Hepato- und/oder Splenomegalie sowie eine Lymphoadenopathie auf einen Immundefekt hinweisen.

Nach AWMF-Leitlinien wird als Basis-Diagnostik bei V. a. auf Immundefekte ein Differenzialblutbild sowie die Bestimmung der Immunglobuline G, A, M und E empfohlen (www.awmf.de). Bei der Bestimmung des Blutbildes ist insbesondere bei Säuglingen und Kleinkindern auf altersentsprechende Normwerte zu achten. Auch diese Laboruntersuchungen sind in der Regel ja Standard in der kinderrheumatologischen Ambulanz und erscheinen relativ simpel im Hinblick auf die Vielzahl der unterschiedlichen Immundefekte. Schaut man sich jedoch die Häufigkeit und Verteilung der verschiedenen Gruppen primärer Immundefekte an ( Abb. 1), so wird klar, dass mit dieser einfachen Labordiagnostik prozentual gesehen schon ein Großteil der Immundefekte identifiziert, bzw. auf das Vorliegen eines solchen ein Hinweis gegeben werden kann (Antikörper-Mangel-Syndrome, ein Anteil der kombinierten Immundefekte, gut definierte Syndrome mit Immundefekt, Defekte der Phagyozten u. a.). Weitere relativ einfache Untersuchungen stellen die Bestimmung spezifischer Antikörper (in der Regel Impfantikörper, z. B. Tetanus und Pneumokokken, Isoagglutinine) und der Lymphozytenpopulationen und ggf. -funktion dar. Je nach Labor können dabei auch Subpopulationen der Lymphozyten wie Gedächtnis-B-Zellen bestimmt werden. Allerdings schließen auch hierbei Normalbefunde einen Immundefekt nicht aus. Die weitere Abklärung sollte dann je nach eigenen Möglichkeiten in Absprache mit einem Immundefektzentrum erfolgen. Die inzwischen zunehmend angebotene Panel-Diagnostik genetischer Erkrankungen bietet auch für die Ambulanz ein gutes Potenzial, molekulargenetische Untersuchungen in begrenztem Maß selbst durchzuführen, z. B. bei kombinierten Immundefekten (CVID) oder der Immundysregulation. Eine vorherige detaillierte klinische Charakterisierung stellt dabei eine wichtige Voraussetzung dar, das richtige Panel auszuwählen und erhöht damit die Möglichkeit einer molekulargenetischen Diagnosefindung. Das Whole-Exom-Sequencing ist wohl weiterhin nur in größeren Zentren möglich.

\section{Therapie von rheumatischen Symptomen bei Patienten mit primären Immundefekten}

Bei Patienten mit bekanntem Immundefekt und Vorliegen einer Arthritis muss in jedem Fall eine infektiöse Ursache ausgeschlossen werden, da dies einen anderen Therapieansatz erfordert. So haben wir z. B. einen Patienten mit einem Cernunnos-Immundefekt mit einer chronischen Arthritis durch eine persistierende Adenovirus-Infektion u. a. mit einer Erhöhung der substituierten Immunglobulin-Gabe behandelt [49].

Bestätigt sich eine autoimmune Genese als Ursache der rheumatischen Beschwerden, so richtet sich das weitere Vorgehen danach, inwieweit der Immundefekt bekannt ist. Liegt keine genetisch gesi- 
$\checkmark$ Tab. 1 PID und rheumatologische Manifestationen.

\begin{tabular}{|c|c|c|c|c|}
\hline & Immundefekt & Mutation & Charakteristische Symptome & $\begin{array}{l}\text { Rheumatologische } \\
\text { Manifestationen }\end{array}$ \\
\hline \multirow[t]{6}{*}{$\begin{array}{l}\text { Humorale } \\
\text { Immundefekte }\end{array}$} & $\begin{array}{l}\text { M. Bruton (X-linked } \\
\text { Agammaglobuline- } \\
\text { mia; XLA) }\end{array}$ & $\begin{array}{l}\text { Bruton's Tyrosin Kinase } \\
\text { (btk) }\end{array}$ & $\begin{array}{l}\text { Bakterielle Atemwegsinfekte, rez. Otitiden, Gira- } \\
\text { dia-lamblia-Enteritis }\end{array}$ & $\begin{array}{l}\text { Arthritis (Oligoarthritis, } \\
\text { enthesitis-ass. Arthritis, } \\
\text { ersoive Polyarthritis) }\end{array}$ \\
\hline & $\begin{array}{l}\text { Selektiver } \\
\text { IgA-Mangel }\end{array}$ & Unbekannt & $\begin{array}{l}\text { Häufig asymptomatisch, sonst rez. Infekte der Atem- } \\
\text { wege, Autoimmunität }\end{array}$ & JIA \\
\hline & $\begin{array}{l}\text { Hyper-IgM-Syn- } \\
\text { drome }\end{array}$ & $\begin{array}{l}\text { CD40L, CD40, AID, } \\
\text { UNG }\end{array}$ & $\begin{array}{l}\text { Rez. Atemwegsinfektionen, opportunistische Infekti- } \\
\text { on mit P. jiruveci und Kryptosporidien }\end{array}$ & Arthritis, SLE \\
\hline & \multirow[t]{3}{*}{$\begin{array}{l}\text { Variabler Immun- } \\
\text { defekt (CVID) }\end{array}$} & $\begin{array}{l}\text { BAFF-R, TACI, BCMA, } \\
\text { APRIL, CD19, CD20, } \\
\text { CD21, in } 90 \% \text { weiter- } \\
\text { hin unbekannt }\end{array}$ & $\begin{array}{l}\text { Infektneigung, granulomatöse Erkrankungen, Au- } \\
\text { toimmunität, Malignome }\end{array}$ & Arthritis, SLE, Vaskulitis \\
\hline & & NF-KB 1 & $\begin{array}{l}\text { Lymphadenopathie, Splenomegalie, Autoimmunsym- } \\
\text { ptome }\end{array}$ & Polyarthritis \\
\hline & & 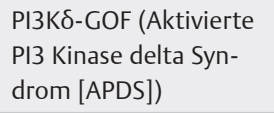 & $\begin{array}{l}\text { Rez. Atemwegsinfektionen, Lymphoproliferation, } \\
\text { Splenomegalie, Autoimmunsymptome }\end{array}$ & Chronische Arthritis \\
\hline \multirow[t]{5}{*}{$\begin{array}{l}\text { Erkrankungen } \\
\text { mit Immun- } \\
\text { dysregulation }\end{array}$} & IPEX-Syndrom & FOXP-3 & $\begin{array}{l}\text { Enterokolitis, Typ-1-Diabetes, Hypoparathyreoidis- } \\
\text { mus, hämolytische Anämie, membranöse Glomeru- } \\
\text { lonephritis }\end{array}$ & Arthritis, Vaskulitis \\
\hline & \multirow[t]{4}{*}{$\begin{array}{l}\text { Andere „Tregopa- } \\
\text { thien“ }\end{array}$} & CTLA-4 Defizienz & $\begin{array}{l}\text { Zytopenien, Lymphoproliferation, Splenomegalie, } \\
\text { Hepatomegalie, Lymphadenopathie, Enteropathien, } \\
\text { atopische Dermatitis, Autoimmunezephaliltis und } \\
\text {-thyreoiditis }\end{array}$ & $\begin{array}{l}\text { Rheumatoide Arthritis, Pso- } \\
\text { riasisarthritis }\end{array}$ \\
\hline & & LRBA-Defizienz & Ähnlich wie CTLA-4 Defizienz & Arthritis \\
\hline & & STAT1-GOF & $\begin{array}{l}\text { Lymphoproliferation, Autoimmunsymptome (Ente- } \\
\text { ropathie, Zöliakie, Diabetes mel. Typ 1, interstitielle } \\
\text { Lungenerkrankung) }\end{array}$ & $\begin{array}{l}\text { Polyarthritis, Sklerodermie, } \\
\text { Uveitis }\end{array}$ \\
\hline & & STAT3-GOF & $\begin{array}{l}\text { Chronisch mukokutane Candidiasis, rez. Atemwegs- } \\
\text { infekte, Herpesinfektionen, invasive Pilzinfektionen, } \\
\text { Hautinfektionen mit S. aureus, Autoimmunsympto- } \\
\text { me (Zytopenien, Hypothyreose, T1DM) }\end{array}$ & $\begin{array}{l}\text { SLE, Vaskulitis, Anti-phophos- } \\
\text { lipid Syndrom, Sklerodermie }\end{array}$ \\
\hline \multirow{2}{*}{$\begin{array}{l}\text { Gut definierte } \\
\text { Syndrome mit } \\
\text { Immundefekt }\end{array}$} & DiGeorge-Syndrom & 22q11 Mikrodeletion & $\begin{array}{l}\text { Herzfehler, Infektanfälligkeit, Dysmorphiezeichen, } \\
\text { Hypokalzämie }\end{array}$ & Polyarthritis \\
\hline & $\begin{array}{l}\text { Wiskott-Aldrich- } \\
\text { Syndrom }\end{array}$ & $\begin{array}{l}\text { Wiskott-Aldrich-Syn- } \\
\text { drom-Protein (WASp) }\end{array}$ & $\begin{array}{l}\text { Rez. Infekte, Ekzem, Thrombozytopenie, Autoimmu- } \\
\text { nität, Malignome }\end{array}$ & Arthritis, Vaskulitis \\
\hline \multirow[t]{2}{*}{ Andere } & $\begin{array}{l}\text { Septische Granulo- } \\
\text { matose }\end{array}$ & $\begin{array}{l}\text { gp91phox, p22phox, } \\
\text { p47phox, p67phox }\end{array}$ & $\begin{array}{l}\text { Rez. Infekte mit Bakterien und Pilzen, Abszesse, chro- } \\
\text { nisch entzündliche Darmerkrankung, Granulome }\end{array}$ & $\begin{array}{l}\text { Arthritis, SLE, diskoider Lupus } \\
\text { (auch bei Überträgerinnen) }\end{array}$ \\
\hline & $\begin{array}{l}\text { Komplementde- } \\
\text { fekte }\end{array}$ & $\mathrm{C} 1 \mathrm{q}, \mathrm{C} 1 \mathrm{r} / \mathrm{C} 1 \mathrm{~s}, \mathrm{C2}, \mathrm{C} 4$ & Schwere bakterielle Infekte & SLE \\
\hline
\end{tabular}

cherte Diagnose vor, so empfiehlt sich das übliche therapeutische Vorgehen wie bei der JIA und anderen rheumatischen Erkrankungen. Gibt es eine genetisch gesicherte Diagnose, kann durch die Vielzahl der inzwischen vorhandenen Biologika versucht werden, gezielt in die Pathogenese einzugreifen. Beispiele dafür sind eine Behandlung mit Abatacept bei der CTLA-4-Defizienz oder JAK-Inhibitoren bei STAT3-GOF-Mutationen. Darüber hinaus haben sich einige Medikamente bei bestimmten Immundefekten als besonders wirksam erwiesen, sodass es sich empfiehlt, diese bei der Wahl der Therapie bevorzugt einzusetzen. Eine Zulassung fehlt jedoch in den allermeisten Fällen. \ Tab. 2 zeigt entsprechende Beispiele für die hier aufgeführten Erkrankungen. Bei einigen primären Im- mundefekten stellt die Stammzelltransplantation einen kurativen Therapieansatz dar, bei dem nach erfolgreicher Durchführung in der Regel auch die Autoimmunsymptome sistieren. Zudem wird die Gentherapie von primären Immundefekten in Zukunft sicherlich eine größere Rolle einnehmen. 
\ Tab. 2 Beispiele für therapeutische Optionen für rheumatologische Manifestationen bei definierten Immundefekten.

\begin{tabular}{|c|c|c|c|}
\hline Primärer Immundefekt & Therapeutikum & Zielstruktur & Wirkmechanismus \\
\hline \multirow[t]{3}{*}{ CTLA-4/LRBA Defizienz } & Abatacept & CD28 & $\begin{array}{l}\text { Fusionsprotein aus Fc-Teil von IgG1 und extrazel- } \\
\text { lulärer Domäne von CTLA-4, bindet an CD80/86 } \\
\text { auf Antigen-präsentierenden Zellen, verhindert so } \\
\text { Interaktion mit CD28 auf T-Zellen }\end{array}$ \\
\hline & Sirolimus (Rapamycin) & m-TOR Signalweg & S6K/m-Tor-Inhibitor \\
\hline & Mycophenolat & Inosin Monophosphat Dehydrogenase (IMD) & Reversibler IMD-Inhibitor \\
\hline \multirow{3}{*}{$\begin{array}{l}\text { Aktivierte PI3 Kinase } \\
\text { delta Syndrom (APDS) }\end{array}$} & Leniolisib & $\mathrm{p} 110 \delta$ & p110ס-Inhibitor \\
\hline & Rituximab & CD20 & $\begin{array}{l}\text { Bindung an CD20 auf B-Zellen, dadurch B-Zell- } \\
\text { Depletion }\end{array}$ \\
\hline & Mycophenolat & Inosin Monophosphat Dehydrogenase & Reversibler IMD-Inhibitor \\
\hline \multirow[t]{3}{*}{ STAT1- und STAT3-GOF } & Ruxolitinib & JAK $1 / 2$ & JAK-Inhibitor \\
\hline & Baricitinib & JAK $1 / 2$ & JAK-Inhibitor \\
\hline & Tofacitinib & JAK $1 / 2$ & JAK-Inhibitor \\
\hline
\end{tabular}

\section{Interessenkonflikt}

Die Autorin gibt an, dass kein Interessenkonflikt besteht.

\section{Literatur}

[1] Picard C et al. International Union of Immunological Societies: 2017 Primary Immunodeficiency Diseases Committee Report on Inborn Errors of Immunity. J Clin Immunol 2018; 38(1): 96-128

[2] Walter JE, Ayala IA, Milojevic D. Curr Opin Pediatr 2019;31(6): 851-862. doi: 10.1097

[3] Fischer A, Provot J, Jais JP et al.; members of the CEREDIH French PID study group. J Allergy Clin Immunol 2017; 140(5): 1388-1393.e8. doi: 10.1016/j.jaci.2016.12.978. Epub 2017 Feb 10. PMID: 28192146

[4] Bruton OC et al. Absence of serum gamma globulins. AMA Am J Dis Child 1952; 84(5): 632-636

[5] Jesus AA., Duarte AJ, Oliveira JB. Autoimmunity in hyper-IgM syndrome. J Clin Immunol 2008; 28 (Suppl 1): S62-S66

[6] Fu JL et al .X-linked agammaglobulinemia presenting as juvenile chronic arthritis: report of one case. Acta Paediatr Taiwan 1999; 40(4): 280-283

[7] Sukumaran $\mathrm{S}$ et al. A child with $\mathrm{x}$-linked agammaglobulinemia and enthesitis-related arthritis. Int J Rheumatol 2011; 2011: 175973

[8] Verbruggen $G$ et al. $X$ linked agammaglobulinaemia and rheumatoid arthritis. Ann Rheum Dis 2005; 64(7): 1075-1078

[9] Levy J et al. Clinical spectrum of X-linked hyper-IgM syndrome. J Pediatr 1997; 131(1 Pt 1): 47-54

[10] Webster EA et al. An aggressive form of polyarticular arthritis in a man with CD154 mutation (X-linked hyper-IgM syndrome). Arthritis Rheum 1999; 42(6): 1291-1296

[11] Sibilia J et al. Hyper-IgM syndrome associated with rheumatoid arthritis: report of RA in a patient with primary impaired CD40 pathway. $\mathrm{Br}$ ] Rheumatol 1996; 35(3): 282-284

[12] Melegari A et al. Immunodeficiency and autoimmune phenomena in female hyper-IgM syndrome. Ann N Y Acad Sci 2007; 1109: 106-108

[13] Jorgensen $\mathrm{GH}$ et al. Familial aggregation of IgAD and autoimmunity. Clin Immunol 2009; 131(2): 233-239
[14] Cassidy JT, Pettym RE, Sullivan DB. Abnormalities in the distribution of serum immunoglobulin concentrations in juvenile rheumatoid arthritis. J Clin Invest 1973; 52(8): 1931-1936

[15] Odnoletkova I et al. The burden of common variable immunodeficiency disorders: a retrospective analysis of the European Society for Immunodeficiency (ESID) registry data. Orphanet J Rare Dis 2018; 13(1): 201

[16] Kuijpers TW et al. CD20 deficiency in humans results in impaired T cell-independent antibody responses. J Clin Invest 2010; 120(1): 214-222

[17] van Zelm MC et al. An antibody-deficiency syndrome due to mutations in the CD19 gene. N Engl J Med 2006; 354(18): 1901-1912

[18] van Zelm MC et al. CD81 gene defect in humans disrupts CD19 complex formation and leads to antibody deficiency. J Clin Invest 2010; 120(4): 1265-1274

[19] Wentink MW et al. CD21 and CD19 deficiency: Two defects in the same complex leading to different disease modalities. Clin Immunol 2015; 161(2): 120-127

[20] Grimbacher B et al. Homozygous loss of ICOS is associated with adult-onset common variable immunodeficiency. Nat Immunol 2003; 4(3): 261-268

[21] Castigli E et al. TACl is mutant in common variable immunodeficiency and IgA deficiency. Nat Genet 2005; 37(8): 829-834

[22] Warnatz $\mathrm{K}$ et al. B-cell activating factor receptor deficiency is associated with an adult-onset antibody deficiency syndrome in humans. Proc Natl Acad Sci U S A 2009; 106(33): 13945-13950

[23] Tuijnenburg $P$ et al. Loss-of-function nuclear factor kappaB subunit 1 (NFKB1) variants are the most common monogenic cause of common variable immunodeficiency in Europeans. | Allergy Clin Immunol 2018; 142(4): 1285-1296

[24] Chen K et al. Germline mutations in NFKB2 implicate the noncanonical NF-kappaB pathway in the pathogenesis of common variable immunodeficiency. Am J Hum Genet 2013; 93(5): 812-824

[25] Conley ME, Notarangelo LD, Etzioni A. Diagnostic criteria for primary immunodeficiencies. Representing PAGID (Pan-American Group for Immunodeficiency) and ESID (European Society for Immunodeficiencies). Clin Immunol 1999; 93(3): 190-197

[26] Elkaim E et al. Clinical and immunologic phenotype associated with activated phosphoinositide 3-kinase delta syndrome 2: A cohort study. | Allergy Clin Immunol 2016; 138(1): 210-218 e9 
[27] Jamee M et al. Clinical, Immunological, and Genetic Features in Patients with Activated PI3Kdelta Syndrome (APDS): a Systematic Review. Clin Rev Allergy Immunol 2019 May 21. doi: 10.1007/s12016-01908738-9

[28] Coulter TI et al. Clinical spectrum and features of activated phosphoinositide 3-kinase delta syndrome: A large patient cohort study. J Allergy Clin Immunol 2017; 139(2): 597-606 e4

[29] Barzaghi F et al. Long-term follow-up of IPEX syndrome patients after different therapeutic strategies: An international multicenter retrospective study. J Allergy Clin Immunol 2018; 141(3): 1036-1049 e5

[30] Gambineri E et al. Clinical, Immunological, and Molecular Heterogeneity of 173 Patients With the Phenotype of Immune Dysregulation, Polyendocrinopathy, Enteropathy, X-Linked (IPEX) Syndrome. Front Immunol 2018; 9: 2411

[31] Cepika AM et al. Tregopathies: Monogenic diseases resulting in regulatory T-cell deficiency. J Allergy Clin Immunol 2018; 142(6): 1679-1695

[32] Mitsuiki N, Schwab C, Grimbacher B. What did we learn from CTLA-4 insufficiency on the human immune system? Immunol Rev 2019; 287(1): 33-49

[33] Schwab C et al. Phenotype, penetrance, and treatment of 133 cytotoxic T-lymphocyte antigen 4-insufficient subjects. J Allergy Clin Immunol 2018; 142(6): 1932-1946

[34] Lopez-Herrera $G$ et al. Deleterious mutations in LRBA are associated with a syndrome of immune deficiency and autoimmunity. Am J Hum Genet 2012; 90(6): 986-1001

[35] Gámez-Díaz L, August D, Stepensky P et al. J Allergy Clin Immunol 2016;137(1): 223-230. doi: 10.1016/j.jaci.2015.09.025

[36] Oz RS, Gluth M, Tesher MS. Pediatric Autoimmune Inner Ear Disease: A Rare, But Treatable Condition. Pediatr Ann 2019; 48(10): e391-e394

[37] Mogensen TH. IRF and STAT Transcription Factors - From Basic Biology to Roles in Infection, Protective Immunity, and Primary Immunodeficiencies. Front Immunol 2018; 9: 3047

[38] Gadina $\mathrm{M}$ et al. Translational and clinical advances in JAK-STAT biology: The present and future of jakinibs. J Leukoc Biol 2018; 104(3): 499-514
[39] Holland SM et al. STAT3 mutations in the hyper-IgE syndrome. N Engl ] Med 2007; 357(16): 1608-1619

[40] Haapaniemi EM et al. Autoimmunity, hypogammaglobulinemia, lymphoproliferation, and mycobacterial disease in patients with activating mutations in STAT3. Blood 2015; 125(4): 639-648

[41] Milner JD et al. Early-onset lymphoproliferation and autoimmunity caused by germline STAT3 gain-of-function mutations. Blood 2015; 125(4): 591-599

[42] Toubiana J et al. Heterozygous STAT1 gain-of-function mutations underlie an unexpectedly broad clinical phenotype. Blood 2016; 127(25): 3154-3164

[43] Pelkonen P et al. Chronic arthritis associated with chromosome deletion 22q11.2 syndrome. J Rheumatol 2002; 29(12): 2648-2650

[44] Sullivan KE et al. Juvenile rheumatoid arthritis-like polyarthritis in chromosome 22q11.2 deletion syndrome (DiGeorge anomalad/velocardiofacial syndrome/conotruncal anomaly face syndrome). Arthritis Rheum 1997; 40(3): 430-436

[45] Crestani E et al. Broad spectrum of autoantibodies in patients with Wiskott-Aldrich syndrome and X-linked thrombocytopenia. J Allergy Clin Immunol 2015; 136(5): 1401-1404 e1-3

[46] Lee BW, Yap HK. Polyarthritis resembling juvenile rheumatoid arthritis in a girl with chronic granulomatous disease. Arthritis Rheum 1994; 37(5): 773-776

[47] Winkelstein JA et al. Chronic granulomatous disease. Report on a national registry of 368 patients. Medicine (Baltimore) 2000; 79(3): 155-169

[48] Schroder-Braunstein J, Kirschfink M. Complement deficiencies and dysregulation: Pathophysiological consequences, modern analysis, and clinical management. Mol Immunol 2019; 114: 299-311

[49] Meyer-Bahlburg A, Dressler F, Baumann U. Chronic arthritis in a boy with Cernunnos immunodeficiency. Clin Immunol 2014; 154(1): 47-48 